Service social

\title{
La rationalité des émotions dans les processus de mobilisation collective
}

\section{Martine Duperré}

Volume 54, numéro 1, 2008

URI : https://id.erudit.org/iderudit/018344ar

DOI : https://doi.org/10.7202/018344ar

Aller au sommaire du numéro

Éditeur(s)

École de service social de l'Université Laval

ISSN

0037-2633 (imprimé)

1708-1734 (numérique)

Découvrir la revue

Citer cet article

Duperré, M. (2008). La rationalité des émotions dans les processus de mobilisation collective. Service social, 54(1), 67-81.

https://doi.org/10.7202/018344ar
Résumé de l'article

Dans la perspective d'identification des facteurs et des processus de mobilisation, plusieurs éléments ont effectivement été reconnus, mais dans cette analyse, les émotions ont d'abord représenté un accroc à la rationalité et ensuite sont totalement évacuées. Basé sur des données empiriques, cet article propose de montrer que les émotions sont une donnée dont il faut tenir compte dans l'analyse des groupes mobilisés, car elles influencent l'engagement, la cohésion, la désagrégation, ainsi que les stratégies utilisées par les acteurs. 


\title{
La rationalité des émotions dans les processus de mobilisation collective
}

\author{
Martine DUPERRÉ \\ Professeure agrégée \\ Ph. D. en Sciences régionales \\ École de service social \\ Université Laval
}

Dans la perspective d'identification des facteurs et des processus de mobilisation, plusieurs éléments ont effectivement été reconnus, mais dans cette analyse, les émotions ont d'abord représenté un accroc à la rationalité et ensuite sont totalement évacuées. Basé sur des données empiriques, cet article propose de montrer que les émotions sont une donnée dont il faut tenir compte dans l'analyse des groupes mobilisés, car elles influencent l'engagement, la cohésion, la désagrégation, ainsi que les stratégies utilisées par les acteurs.

Mots-clefs: mobilisation, action collective, organisation communautaire, émotions, santé mentale.

In the perspective of the identification of factors and mobilizing processes, many elements were in fact identified by analysts. Unfortunately, in this analysis, emotions represent a problem. In some theorizing they were first considered as a rationality problem and in the next generation they were completely evacuated. Based on empirical data, this paper will demonstrate that emotions are very important in the mobilizing processes as they influence individual engagement, group cohesion and collective actor strategies.

Key words: mobilization, collective action, community practice, emotions, mental health.

\section{INTRODUCTION}

L'histoire de toutes les sociétés et de tous les temps est jalonnée de mobilisations menées par des acteurs collectifs qui contestent certains aspects de l'organisation sociale et qui proposent un nouveau projet collectif pour une société plus juste à leur endroit. Par cette action, ils participent à la structuration de la société (Giddens, 1987). Depuis de nombreuses années, les chercheurs tentent de déterminer les facteurs et les processus de mobilisation et de constitution de ces mouvements sociaux et de ces acteurs collectifs. Plusieurs facteurs ont effectivement été identifiés, mais dans cette 
analyse, les émotions ont toujours posé un problème lié à la rationalité supposée de l'acteur, soit qu'elles étaient considérées comme un accroc à la rationalité, soit qu'elles étaient complètement évacuées au profit d'un acteur dont la rationalité supposée était uniquement instrumentale. II en résulte que depuis les années 1960, les émotions n'ont pratiquement pas joué de rôle dans les théories sur les mouvements sociaux et l'action collective (Goodwin, Jasper et Polletta, 2001). Or, sans nier qu'à certaines occasions les émotions peuvent perturber la raison, elles ne sont pas non plus un empêchement systématique à la pensée rationnelle, au contraire, comme l'a montré Damasio (2006). Les émotions permettent aux acteurs d'acquérir connaissance et compréhension des situations; elles sont nécessaires à tout processus interprétatif (Gould, 2004). Par extension, elles sont utiles à la prise de décision de se mobiliser ou pas, et président au choix des stratégies et des tactiques utilisées par l'acteur collectif. En ce sens, on peut considérer rationnelle une décision qui tient compte des émotions, et le rôle de celles-ci doit alors être mieux connu dans le contexte des groupes engagés dans l'action collective.

Le présent article expose les premiers résultats d'une recherche en cours. II se propose d'analyser et de comprendre le rôle des émotions dans les processus de mobilisation des acteurs collectifs. À l'instar de Lamoureux, Lavoie, Mayer et PanetRaymond (2002), la mobilisation est vue ici sous l'angle du regroupement des personnes touchées par un problème social et de leur engagement dans un projet permettant de le résoudre ou à une manière de se lier à d'autres pour l'action collective (Havard-Duclos et Nicours, 2005). Cette définition ne pose pas la mobilisation comme synonyme d'une lutte collective, qu'elle soit à grand déploiement ou non. Elle suppose uniquement l'engagement dans un regroupement qui travaille à résoudre un problème social. Plus précisément, le processus de mobilisation sera regardé à travers trois aspects de l'action collective: celui des trajectoires biographiques individuelles qui amènent certaines personnes à s'engager dans l'action; celui des fonctionnements collectifs internes d'une association de groupes communautaires; et celui des rapports qu'entretient cette association avec d'autres acteurs, particulièrement les organisations gouvernementales. À partir d'un corpus empirique extrait d'une recherche plus large dans le champ de la santé mentale, il sera montré que les émotions sont une donnée dont il faut tenir compte dans l'analyse et dans l'intervention auprès des groupes mobilisés, car elles influencent l'engagement, la cohésion, la désagrégation et les stratégies utilisées par les acteurs. Avant de procéder à cette démonstration, les théories sociologiques sur l'action collective seront exposées afin d'analyser la place occupée par les émotions dans les explications qu'elles font de l'action collective. Ensuite, une définition du concept d'émotion sera proposée. Suivra une description de la méthodologie utilisée dans le projet de recherche général et la manière dont les données ciblées ont été extraites. Enfin, les données empiriques montreront l'importance de tenir compte des émotions dans les processus d'action collective ainsi que l'implication de ce constat pour la recherche et l'intervention dans ce domaine. 


\section{L'ANALYSE DES ÉMOTIONS DANS LES ÉCRITS SCIENTIFIQUES : DE HULK À M. SPOCK}

On peut distinguer trois traditions dans l'analyse des mobilisations collectives. La première est celle de la psychologie des foules et du comportement collectif (dernier quart du XXI ${ }^{\text {ème }}$ siècle). Les auteurs de cette tradition sont Le Bon, Tarde et Taine, et ils postulent que l'individu s'engage dans une action collective en fonction de pulsions irrationnelles et même parfois pathologiques. Pour cette tradition, la foule est caractérisée par «le faible contrôle des affects et des instincts qui la rend émotive, imprévisible et dangereuse» (Neveu, 1996). Ainsi, les manifestants sont considérés comme des êtres anormaux, irrationnels, des déviants émotionnels qui menacent l'ordre social (Aminzade et McAdam, 2001), qu'on pourrait comparer à Hulk, célèbre personnage vert des bandes dessinées qui ne peut exprimer sa colère selon un schéma acceptable. Ce point de vue sur la foule est celui des élites sociales au lendemain de la commune de Paris (Barrows, cité dans Neveu, 1996), ce qui fait dire à Calhoun (2001) que c'est une « approche de l'extérieur» (p. 48), c'est-à-dire l'étude par des analystes d'un phénomène qui ne leur est pas sympathique, auquel ils ne participeraient pas. C'est l'inverse dans la tradition suivante, celle de la théorie de la mobilisation des ressources (TMR).

La TMR est une tradition américaine qui postule que la mobilisation survient lorsque que les participants potentiels estiment qu'une action collective a de fortes chances de réussite, après avoir fait un calcul des différences entre les coûts et les bénéfices supposés. Cette théorisation s'inscrit en réaction aux postulats antérieurs des auteurs de la psychologie des foules et affirme une action collective rationnelle en tombant parfois dans l'excès inverse des théories précédentes. Les émotions y sont évacuées complètement (Marx-Ferree et Merril, 2004). L'acteur, comparable à un M. Spock collectif, décide de son action en fonction de la seule et unique logique rationnelle de calcul coûts/bénéfices, dont la réponse conditionne sa mobilisation.

Certains auteurs associés à ce courant soutiennent maintenant qu'on n'a pas pris en compte que les émotions pouvaient en elles-mêmes motiver à l'action, sans nécessairement entrer dans le calcul coûts/bénéfices (Aminzade et McAdam, 2001). La réduction de la motivation humaine à cette seule dimension du calcul rationnel s'explique par plusieurs raisons. Premièrement, dans une société où la rationalité froide est valorisée au détriment de l'expression des émotions et parce que les acteurs des mouvements sociaux veulent être pris au sérieux, il est normal qu'ils décrivent ainsi leur motivation (Aminzade et McAdam, 2001; Dobbin, 2001). Deuxièmement, Aminzade et McAdam (2001) avancent la thèse que les perspectives analytiques ont pu être teintées par le genre et la position idéologique des analystes, qui étaient majoritairement des hommes nord-américains « [...] qui ont appris, dans leur culture, à minimiser ou réprimer les émotions dans leur vie personnelle et académique » (p. 23) et qu'ils étaient sympathiques aux mouvements et qu'en conséquence, ils voulaient les présenter sous un jour favorable. Enfin, ils appuient également l'idée que les méthodes permettant de saisir les émotions dans l'analyse des mouvements sont peu utilisées, 
parce que selon l'épistémologie positiviste, les émotions déforment l'observation et entravent la connaissance.

Deux voies particulières de la TMR méritent qu'on s'y attarde. Premièrement, il y a le courant du processus politique qui soutient que la mobilisation est rendue possible si un système politique est favorable à l'intégration des revendications des acteurs; c'est ce qui est nommé l'ouverture du système politique. Or, ce courant n'explique pas la montée d'un mouvement malgré la fermeture des opportunités, comme c'était le cas lors des manifestations étudiantes de la place Tien An Men en Chine, ou, inversement, le déclin malgré une plus grande ouverture (Gould, 2004). Pour cela, il faut avoir recours aux émotions mobilisatrices.

Deuxièmement, on doit s'attarder à la framing analysis ou théorie des cadres (Benford et Snow, 2000) qui considère l'action collective comme le résultat du processus d'ajustement des cadres cognitifs, soit les cadres diagnostique (ce qui pose problème), pronostique (comment le résoudre) et motivationnel. Bien que les auteurs du courant soient assez prolixes sur les processus de cadrage diagnostique et pronostique, ils le sont fort peu lorsque vient le moment d'expliquer le cadrage motivationnel, qui est le concept idéal si on veut considérer les émotions dans l'analyse.

Cette brève revue des écrits scientifiques se termine par la perspective européenne des nouveaux mouvements sociaux (NMS), qui souligne l'importance de la nature culturelle de ces derniers et celle de la production de significations et d'identités culturelles. Les NMS luttent pour le contrôle des modes de production de significations et la constitution des identités collectives. Cette perspective analytique met l'accent sur les aspects expressifs des mouvements sociaux et les place exclusivement sur le terrain de la société civile, cette dernière étant opposée à l'État (Canel, 1992). Dans cette approche appelée parfois paradigme identitaire, on met l'accent presque exclusivement sur les processus cognitifs. II existe cependant une exception amenée par Alberto Melucci (1994), pour qui il ne peut avoir de cognition sans sentiments.

\section{COMMENT COMPRENDRE LE CONCEPT «ÉMOTION »}

En 1978, Théodore Kemper (cité dans Goodwin, Jasper et Polletta, 2001) a défini les émotions comme étant « une réponse évaluative relativement à court terme essentiellement positive ou négative, impliquant des composantes somatiques (et souvent cognitives) » (p. 10). Cette définition fait l'économie de dire que les émotions sont aussi des significations sociales partagées, au contraire d'un état physiologique automatique, et font partie de la culture autant que la cognition ou les visions morales (Jasper, 1998). Bien qu'il y ait place pour des variations individuelles ou même de la déviance, les sentiments appropriés à des circonstances particulières sont généralement appris par socialisation, comme le sont d'autres manières de faire propres aux cultures locales.

Une émotion est un sentiment, un affect, c'est-à-dire un état d'esprit élémentaire culturellement délimité, qui n'a de sens que dans des circonstances particulières. Pour 
Aminzade et McAdam (2001), cinq dimensions peuvent être attribuées aux émotions. Ils empruntent les quatre premières à Peggy Thoits (1989) et la cinquième à Michelle Rosaldo (1984). La première dimension est cognitive et consiste en l'évaluation d'un stimulus ou d'un contexte. La deuxième est celle des changements corporels amenés par cette évaluation. La troisième est l'expression de l'émotion par des gestes. La quatrième consiste à étiqueter, selon le schéma culturel en cours, une composition de deux ou trois des premières dimensions, c'est l'interprétation. Enfin, la cinquième dimension, nommée l'engagement, amène l'idée que l'engagement profond de l'acteur dans une situation donnée module sa réponse émotionnelle. Par exemple, une personne n'aura pas la même réponse émotionnelle aux pleurs d'un bébé, selon que celui-ci est le sien ou non.

Les émotions incluent les affects définis par Jasper comme des sentiments plus durables, par exemple, l'amour pour la famille, le sentiment d'identification à un groupe, la loyauté, l'affection d'un lieu et des objets, etc. Les affects peuvent influencer la manière d'agir des gens. Par exemple, si un quartier a des connotations positives de sécurité et de calme, on pourrait prédire qu'un résidant va lutter pour conserver ce caractère (Jasper, 1998).

Jasper propose deux catégorisations d'émotions. Dans la première catégorisation, on compte d'abord les émotions réactives, qui sont des réponses temporaires, et ensuite les affects, qui sont des réponses plus durables et profondes (Aminzade et McAdam, 2001; Jasper, 1998). Dans la deuxième catégorisation de Jasper, les émotions sont dites réciproques ou partagées. Les émotions partagées sont celles que vivent tous les membres d'un groupe envers une situation extérieure. À titre d'exemple, on peut nommer la crainte que pourraient ressentir les membres des groupes communautaires face à une réorganisation du réseau institutionnel de prestation des services sociosanitaires. La dynamique des émotions à l'extérieur du mouvement peut pousser une personne à se joindre au mouvement, participer à un événement, contribuer financièrement ou à être réceptive au plaidoyer d'un leader (Jasper, 1998).

Une émotion est réciproque lorsqu'elle est vécue entre les membres d'un groupe donné. On peut parler ici d'affection, d'amour ou de tension. Les émotions partagées soutiennent les mouvements dans les phases moins actives (Duperré, 2002; Jasper, 1998). Les émotions partagées vont renforcer les émotions réciproques, parce que les personnes ressentent la même chose. À l'inverse, l'affection mutuelle crée un contexte fertile pour l'émergence des émotions partagées. Les deux sortes d'émotions collectives nourrissent la solidarité, l'identité et la culture du mouvement (Jasper, 1998).

Les émotions sont importantes pour la mobilisation, même si on ne peut affirmer qu'elles sont la cause des mouvements sociaux et des mobilisations. Inversement, les seuls facteurs favorables ne produiraient pas de mouvement en l'absence d'émotions (Aminzade et McAdam, 2001). 


\section{MÉTHODOLOGIE}

Les données faisant l'objet de cet article sont extraites d'une recherche ayant pour but d'identifier les facteurs et les processus de mobilisation des acteurs collectifs auprès de deux grandes associations de groupes communautaires des domaines de la santé mentale et celui des groupes de femmes. Rappelons que la mobilisation est comprise ici comme l'engagement des personnes représentantes d'un groupe communautaire membre, dans la vie associative d'un regroupement qui mène des actions collectives, qu'elles soient ou non des luttes.

Six objectifs spécifiques sont poursuivis par la recherche dont celui de l'identification du rôle des émotions dans les diverses phases de la mobilisation. Pour atteindre ces objectifs, la stratégie de recherche est qualitative par études de cas et par comparaison inter-site (Huberman et Miles., 1991). En effet, la recherche qualitative est particulièrement efficace pour comprendre les processus sociaux et les phénomènes complexes, pour saisir le sens ou le point de vue des acteurs face à leurs actions, et pour mieux comprendre les sentiments, les émotions et les représentations élaborées (Denzin et Lincoln, 1998; Deslauriers, 1991; Deslauriers et Kérésit, 1997).

La totalité des données sera constituée de 50 entrevues semi-dirigées, 6 séances d'observation et plusieurs centaines de sources documentaires couvrant ensemble environ 30 ans de vie associative. L'échantillon est intentionnel et contrasté, c'est-àdire que nous avons choisi deux associations en fonction de certains critères: continuité avec une recherche précédente pour l'une et discontinuité pour l'autre association; avoir plus de 30 groupes membres; avoir des membres répartis sur des territoires géographiques relativement excentrés.

À l'intérieur de ces associations, les critères de choix des personnes interviewées sont: être une personne fondatrice, avoir siégé au conseil d'administration à des périodes clefs de l'histoire de l'association, représenter un organisme membre contemporain ou démissionnaire, être une personne significative identifiée par d'autres interviewées et couvrir toutes les zones géographiques de l'association. Puisqu'une étude de cas est une analyse approfondie d'une situation à l'intérieur de certaines frontières, le chercheur tente de recueillir toutes les informations possibles à partir de plusieurs points de vue. C'est pour cette raison qu'une personne choisie pour être interviewée ne répond pas nécessairement à toutes ces caractéristiques, mais au moins à une d'entre elles.

Les données présentées ici ont été extraites de vingt des cinquante entrevues prévues et concernent exclusivement les groupes du secteur de la santé mentale et l'objectif de comprendre le rôle des émotions dans le processus de mobilisation. Ces vingt entrevues ont été réalisées entre les mois de mars 2006 et mars 2007.

Les entrevues sont enregistrées et transcrites intégralement. Ensuite, elles sont retournées aux personnes interviewées pour être vérifiées, complétées si nécessaire et validées. Elles sont ensuite codées par unité de sens (Deslauriers, 1991; Lofland et 
Lofland, 1984) et ces codes sont alors regroupés verticalement et ensuite horizontalement à l'aide de matrices d'analyse ${ }^{1}$.

Considérant la difficulté qu'éprouvent plusieurs répondants à donner un sens à leur action à partir d'énoncés manifestes des émotions, les expressions et les gestes qui évoquent ces émotions sont aussi considérés et codés. Par exemple, lorsqu'une répondante exprime qu'à l'occasion d'une victoire " on se donne des bines sur le $\operatorname{bras}^{2}$ », l'émotion correspondante était codée «geste de solidarité, joie ». Cela constitue la principale limite de cette analyse, car l'émotion est alors supposée par la personne qui effectue le codage à partir d'un énoncé.

L'échantillon des vingt entrevues revêt les caractéristiques suivantes. II est constitué exclusivement de salariés de groupes communautaires, dont sept hommes et treize femmes, qui représentent leurs organismes au sein d'une association dans laquelle ils sont engagés. En regroupant le nombre d'années de présence au sein de l'association, on constate deux groupes distincts: les membres qui ont une connaissance de l'association d'environ de dix à vingt ans (dix personnes), et ceux qui se sont joints plus tard et qui connaissent l'association depuis moins de dix ans (dix personnes). Parmi ces derniers, cinq personnes interviewées connaissent l'association depuis moins de cinq ans.

\section{LES ÉMOTIONS COMME FACTEUR DE MOBILISATION}

La présentation des constats se fait selon trois lignes directrices: les trajectoires biographiques individuelles; les fonctionnements collectifs de l'association; et les rapports de l'association et de ses membres avec d'autres acteurs, principalement des établissements du réseau institutionnel. De manière générale, on peut dire que les émotions sont des médiateurs présents dans toutes les dimensions et à toutes les étapes de l'action collective.

\section{LES TRAJECTOIRES BIOGRAPHIQUES}

Le terme «trajectoire » traduit l'idée du parcours de vie d'une personne. II y a deux types de trajectoires: celle, unique, des personnes; mais aussi celle, collective, des individus façonnés par des cultures, des événements historiques, des positions dans la structure sociale, par exemple, le fait d'être une femme (Havard-Duclos et Nicours, 2005).

À partir de l'analyse des données recueillies, on peut affirmer d'abord que les traits de la personnalité ou les valeurs engendrent des émotions qui peuvent expliquer l'engagement. Une répondante parlera d'un lien «presque mystique » entre ses

1. Selon Huberman et Miles (1991), ces matrices sont des « table[s] synthétique[s] (matrice, tableau, liste de contrôle) ou une figure. Les entrées sont également multiformes: petits paragraphes, citations, phrases courtes, estimations, abréviations, symboles, etc. » (p. 145).

2 Une « bine » est un léger contact fait avec le poing sur le bras de l'interlocuteur. Le geste est souvent emphatique. 
valeurs personnelles et le travail à faire dans l'organisme $(18)^{3}$. Une autre dira que sa passion du communautaire est engendrée par l'espace créatif qu'il lui permet, et ce, en lien avec sa personnalité. Par exemple :

Au niveau communautaire, c'est une passion. J'ai travaillé au niveau public, avant, mais quand on veut faire un changement [...] c'est plus long [...]. Et moi, je suis créative. J'aime développer, j'aime créer, alors le milieu communautaire, pour moi, c'est mon milieu. J'apprécie comment ça fonctionne, la possibilité de faire des changements rapides, d'améliorer des choses, d'être plus près des besoins des gens. C'est ce que j'aime du communautaire (114).

C'est à partir de tels énoncés qu'on peut comprendre que ces personnes puissent être engagées dans une action collective menée par leur association pour défendre les valeurs et les services offerts dans les organismes communautaires.

Un deuxième constat est que plusieurs personnes ont été profondément choquées par l'aspect totalitaire de l'asile et du domaine, et c'est cela qui les amène à s'engager dans l'action collective. L'une est découragée à l'idée de la permanence du diagnostic, c'est-à-dire qu'une fois étiquetée avec un diagnostic, « on s'en sort jamais » (12). Pour une autre, c'est la « violence institutionnalisée » constatée lors d'un travail dans un institut psychiatrique qui explique l'engagement :

J'étais [titre d'emploi] à l'asile en [période]. Avant j'avais lu ce qu'Erving Goffman avait dit des institutions totalitaires. Donc, milieu avec une violence institutionnalisée, tout ça... Et je le vivais live à ce moment-là, car je voyais les rapports de pouvoir, les petites violences qui étaient exercées sur les bénéficiaires (15).

Ces émotions violentes suscitées par le contact avec le monde des asiles et de la maladie mentale provoquent un engagement fort et supposent la mise de l'avant d'un projet communautaire ou antipsychiatrique. Cette caractéristique a été observée chez les fondateurs, mais chez aucun membre associé plus récemment. Chez ces derniers, c'est plutôt le domaine de la santé ou de la maladie mentale qui provoque des émotions intenses. On parlera d'être « tombé en amour avec la problématique » (13) et même d'avoir eu un " coup de foudre » et que « Depuis ce temps-là, [on est] encore atteint de dépendance à la cause! » (118). Chez ces salariées, on constate un engagement fort dans le travail thérapeutique, mais qui ne se traduit pas toujours par un engagement dans l'action collective.

En conclusion de cette section, on peut avancer que la passion pour le monde communautaire comme mode de prestation des services ou le fait d'avoir été choqué par les conditions de vie des personnes atteintes de maladie mentale amènent à un

3. Les numéros entre parenthèse indiquent l'entrevue de référence. Le premier chiffre identifie le regroupement 1 et les chiffres suivants précisent le numéro séquentiel donné aux entrevues.

4. Tout contenu permettant d'identifier une personne interviewée est retiré des entrevues afin de préserver l'anonymat. 
plus grand engagement dans l'association, comparé à celui des personnes qui sont plus touchées par les individus vivant la problématique.

\section{LES ÉMOTIONS ET LEUR IMPACT SUR LA COHÉSION DE L'ASSOCIATION}

Nous considérons ici les émotions en tant que facteur de cohésion ou de désagrégation de l'association.

\section{Les émotions comme facteur de désagrégation : les tensions}

Considérant l'espace dont nous disposons et bien qu'il existe plusieurs ordres de tensions, nous présentons les deux types qui nous apparaissent les plus conséquents sur la cohésion de l'acteur collectif.

\section{Les tensions sur les fins}

Depuis la fondation de l'association, deux objectifs sont en tension. Le premier est de regrouper les organismes en vue de créer un poids pour l'action collective, ce qui en constitue le but politique. Le deuxième objectif est de fournir du support aux salariés des groupes qui travaillent avec un problème social jugé lourd et difficile, dans un environnement perçu tout aussi lourd et difficile et qui provoque un certain épuisement chez les coordonnateurs de ces organismes.

Lorsque l'aspect de l'entraide prend le dessus dans l'association, les tenants de l'action politique semblent impatients et ils s'inquiètent du moment où «les vieux » seront partis. L'association fera-t-elle encore de l'action collective ? Du même souffle, on exprime la fierté des actions politiques réalisées et l'espoir dans celles qui se dessinent à l'horizon. Ces deux émotions positives amènent un plus grand sentiment d'appartenance au regroupement et une plus grande cohésion.

Inversement, lorsque l'action politique du regroupement se fait plus présente, ceux qui préfèrent les objectifs de support et d'entraide sont moins mobilisés et souvent, se retirent des réunions. Ils ressentent une lassitude certaine pour ces batailles, qui, selon eux, ne changent pas leur quotidien. Par contre, leur enthousiasme est palpable pour les activités du regroupement en lien avec l'intervention psychosociale. L'un dira même que la préoccupation du regroupement pour le côté humain aide à la cohésion.

\section{Les tensions liées au financement}

Pour les groupes communautaires, les ressources sont rares. Cet aspect peut amener une certaine compétition entre les groupes lorsqu'on annonce de nouvelles sommes à être investies dans le financement des groupes. Ce fut le cas pour l'association en cause ici et cette compétition a amené des émotions fortes entre les groupes: méfiance, colère, jalousie, frustration. Plusieurs conséquences peuvent être observées. La première est la mise en place par l'association de balises de fonctionnement plus claires, c'est-à-dire une plus grande structuration de l'acteur collectif. D'ailleurs, une répondante parle de ces tensions en termes de «tensions créatrices » (11). Deuxièmement, on peut constater une certaine démobilisation à la 
suite de ces conflits: "On était dans une phase active quand il y a eu beaucoup d'argent. Ça a joué dur. Ça a joué très très dur. Là, on a pu voir des games spéciales. Ça nous a refroidis un petit peu, ça aussi. On a un peu pris nos distances, chacun ses affaires » (119). Le temps aidant, les groupes qui se retirent redeviennent plus actifs par la suite.

\section{Les émotions comme facteur de cohésion interne}

L'association est un lieu de support, un lieu de solidarité forte, « qui permet de mettre [ses] tripes sur la table », qui apporte du soutien aux membres vivant des moments professionnels difficiles (118) et qui sert de « soupape émotionnelle » (116). Pourtant, ces activités de support mutuel ne sont pas mobilisatrices pour tous. Ainsi, un répondant soutient que «les réunions sont parfois un palmarès des douleurs, c'est parfois plate » (15). Pour contrer cet effet démobilisateur, l'association a formalisé une procédure au début des réunions, où les membres présentent les actions dont ils sont fiers $(114 ; 13)$. Une interviewée souligne que cet ajout permet de sentir la solidarité, qu'il rend le regroupement plus intéressant, parce que les gens se sentent plus engagés en y prenant la parole (14).

Le sentiment de solidarité se traduit en gestes concrets, tels que du support offert à des personnes affectées par des difficultés professionnelles. À ce sujet, on a dit: « c'est une action d'affection, car parfois dans le communautaire, c'est très laid, c'est pas correct! » (11). À d'autres moments, le support est en lien avec la vie personnelle : anniversaires, décès, etc.

L'expression de la solidarité a un impact positif important sur la cohésion. Dans les entretiens, on affirme que cela crée un sentiment d'être une grande famille (110), que les attentions délicates sont bien importantes (111), qu'elles augmentent beaucoup la cohésion au sein du regroupement (116; 16), qu'elles rapprochent les membres (17), qu'elles augmentent le sentiment d'appartenance et que cela donne ensuite le goût de s'investir $(13 ; 11)$, et enfin qu'elles augmentent le sentiment de force (14).

L'humour a aussi une fonction bien importante. II rend les réunions agréables et motive à y assister (111), il aide à passer à travers des moments difficiles (15), et il est utilisé comme tactique dans l'affrontement avec le réseau institutionnel. En effet, les parodies sur le réseau institutionnel servent d'exutoire et à construire une manière commune de voir les choses (15).

L'idée de l'humour nous amène aux activités festives ayant cours au sein de l'association et de leur impact sur la mobilisation. Les fêtes permettent aux gens de s'apprivoiser, ce qui est nécessaire aux discussions ultérieures et pour éviter des conflits qui pourraient survenir (111). Les fêtes mettent le plaisir d'être ensemble à l'ordre du jour $(110,13)$, elles font sortir « d'une sorte d'aigreur par rapport à la somme de travail qu'on peut avoir à l'association » (111). Les fêtes permettent de rencontrer les amis (110) qu'on a pu se faire au gré des années de membership au sein du regroupement. 
L'amitié est un sentiment important à considérer dans la vie de l'association ciblée par notre étude. Pour plusieurs, l'amitié s'est développée au fil du temps et motive la poursuite du membership $(110 ; 111 ; 116 ; 16)$. Pour d'autres, au contraire, elle inquiète, car elle peut influencer la répartition du financement, ou bien une personne peut acquérir plus de pouvoir en étant liée avec des leaders (111; 116).

Dans les derniers mois de l'année 2006, l'association a effectué un retournement important, qui s'est concrétisé en un changement de nom, et ce changement a été très significatif. Alors que l'ancien nom déterminait la place du regroupement en rapport avec leur opposant, le nouveau nom recentre l'identité sur soi, sur un projet à construire. Ce nouveau nom, cette nouvelle orientation apporte une grande fierté, un espoir immense et un emballement nouveau pour l'association $(11 ; 15 ; 17 ; 19)$.

\section{LES ÉMOTIONS À LA BASE DES CHOIX STRATÉGIQUES ET TACTIQUES DE L'ASSOCIATION}

Dans cette section, l'association est considérée dans ses rapports avec le réseau institutionnel de la santé et des services sociaux.

Dès le départ, la peur ou le sentiment de menace ont été une raison pour créer l'association. D'abord, on redoutait ces gros établissements et les résultats de l'affrontement entre des petits groupes isolés contre ce Goliath de la santé mentale (15) dans une dynamique où la restructuration des services semble se concrétiser. Bien plus tard, lors de la mise en place des réseaux cliniques (dernière réforme du réseau de la santé et des services sociaux - 2003), les groupes ont resserré les liens entre eux, car ils craignaient encore que cette nouvelle configuration des services augure mal pour leurs intérêts (19). Ainsi, on peut constater que la fluctuation du sentiment de menace explique les variations de la cohésion au sein de l'association.

Les tensions entre le regroupement et les représentants du réseau institutionnel sont palpables, et la relation avec cet « autre » est identifiée comme une relation « bipolaire » par un répondant (15) ou qualifiée de « relation amour/haine » par un autre (17). C'est dire que les émotions sont à l'ordre du jour et que cette relation ne laisse pas indifférente!

Les répondants arrivent à cette description générale des relations avec le réseau institutionnel, après avoir évoqué l'espoir d'obtenir des gains, c'est-à-dire de se faire reconnaître par l'institutionnel, et la déception face aux résultats : ces deux émotions ont déterminé la stratégie utilisée par le regroupement. Lors de la période d'espoir, on a opté pour une stratégie politique de participation aux instances de concertation. La déception a accompagné le recentrage identitaire et le retrait des personnes siégeant aux lieux de concertation. En effet, on perçoit toutes les difficultés rencontrées dans les relations avec le réseau institutionnel comme un manque de reconnaissance du travail accompli par les groupes. Et vu qu'on ne peut, malgré tous les efforts, se faire reconnaître par l'autre, la revalorisation de soi et du projet porté prend alors tout son sens. 


\section{CONCLUSION}

Ce texte a montré que les émotions intervenaient dans plusieurs aspects de l'engagement dans une association vouée à l'action collective et qu'elles étaient, par extension, un facteur primordial à considérer. D'abord sur le plan individuel, le fait d'avoir été témoin de l'aspect aliénant ou totalitaire de l'asile a déterminé l'engagement de plusieurs personnes de cette association, car cela a suscité des émotions puissantes de colère et de révolte et a donné sens à l'engagement. Ces résultats sont comparables à ceux issus d'une recherche précédente (Duperré, 2002) et montrent bien que quête individuelle de sens et engagement social vont de pair. Cela implique que dans les processus de mobilisation, on doit porter une attention particulière aux trajectoires biographiques afin de répondre aux besoins liés à la quête de sens de la personne à mobiliser.

Considérant la désinstitutionnalisation relativement importante que vit le Québec depuis plusieurs années, on peut poser l'hypothèse que c'est l'importance de la présence de services communautaires qui motive l'engagement des jeunes travailleurs des ressources communautaires.

D'autres émotions, comme on a pu le voir, sont à la base de la mobilisation de l'association. Ainsi, la peur et le sentiment de menace sont aussi de puissants facteurs au début de la mobilisation. Cependant, selon Jasper (1998), on ne pourrait dire qu'une émotion particulière se traduit toujours en un seul type de comportement. Par exemple, la peur est mobilisatrice. Pourtant, poussée à un certain degré, la peur peut être paralysante. De plus, la peur peut servir de motivation principale pour l'action collective dans certains cas, mais elle n'est pas la seule à intervenir. On a aussi montré, à l'instar d'Aminzade et McAdam (2001), que l'espoir devait être présent pour initier le passage à l'action. Ces résultats soulignent aussi l'importance, lors des processus de cadrage de l'action collective - c'est-à-dire les processus qui mènent à une compréhension collective de l'ensemble des éléments de l'action -, de cadrer les émotions: ce qui est injuste et l'espoir de le changer, entre autres (Aminzade et McAdam, 2001).

Les émotions sont aussi présentes dans les dynamiques internes des groupes mobilisés. On a pu remarquer que les émotions réciproques d'amitié et d'affection entre les membres étaient très importantes et qu'elles maintenaient la cohésion du groupe, alors que les événements étaient susceptibles de pousser à la désagrégation, comme ce fut le cas pour le partage des subventions. Dans d'autres cas, ces sentiments permettront de garder l'intégrité du groupe alors que se referme la fenêtre d'occasion permettant l'action collective, ou qu'il n'y a plus d'opposant principal à affronter (Duperré, 2002).

En plus d'aider à détendre l'atmosphère et surmonter des moments difficiles, l'humour peut servir à la construction des cadres de représentations collectifs nécessaires à la mobilisation. Par l'humour, on montre ce qui convient de faire et ce qui ne convient pas, les valeurs qui sont chéries et celles qui sont honnies. L'humour peut aussi servir 
à la socialisation émotionnelle, c'est-à-dire l'apprentissage des règles émotionnelles appropriées au groupe et leur vocabulaire (Aminzade et McAdam, 2001). Ce travail émotionnel permet à l'ensemble des groupes membres d'intégrer la culture propre du collectif et crée ainsi une plus grande cohésion. L'humour, enfin, identifie clairement l'« autre », l'adversaire visé par l'action collective. Selon Aminzade et McAdam (2001), les bons organisateurs savent que l'opposition renforce la cohésion, le sentiment du «nous» et du « eux », et qu'il y a une grande charge émotive qui y est associée.

En résumé, le facteur émotionnel doit absolument être considéré en relation avec les autres facteurs, soit les conditions présentes dans le système d'action (acteurs et facteurs) ou à l'intérieur même du groupe mobilisé, tant dans l'analyse de ces groupes que lors de l'intervention auprès de ceux-ci. Pourtant, les émotions sont difficiles à saisir, elles sont prises pour acquises, elles ne sont pas évidentes. Et quand on leur pose des questions, les répondants ont souvent tendance à favoriser le cognitif, puisque c'est la dimension la plus valorisée dans notre société et qu'ils veulent être pris au sérieux. Cela nécessite certains ajustements à la méthodologie de la recherche à privilégier. Pour la recherche sur les mouvements sociaux et l'action collective, Aminzade et McAdam (2001) suggèrent une approche pluraliste, qui intègre, notamment, les entrevues, les narrations personnelles, du matériel biographique ou autobiographique, et des récits de vie. II ne faut pas oublier l'observation participante, qui demeure, selon Goodwin, Jasper et Poletta (2001), un des meilleurs moyens de saisir les émotions, même si on tient compte que l'observateur doit questionner constamment la justesse de ses perceptions et de ses interprétations. Quoi qu'il en soit, un raffinement méthodologique est à construire dans l'étude et l'analyse des émotions dans les mouvements sociaux et l'action collective, car il y a encore de nombreuses questions qui demeurent sans réponse.

Sur le plan de l'intervention en organisation communautaire, on ne saurait trop décourager une intervention froide, qui ne tiendrait pas compte, tant des émotions pouvant amener les personnes à s'engager dans l'action collective, que des réalités émotionnelles vécues par les membres pendant les actions menées par le groupe mobilisé. Qui n'a pas ressenti l'énergie de la solidarité vécue dans les grandes mobilisations ? Qui n'a pas constaté le découragement de certains quand une victoire était longue à venir ? Enfin, le facteur émotionnel est peut-être aussi celui qui explique les reculs constatés et dont on saisissait mal le pourquoi. 


\section{RÉFÉRENCES BIBLIOGRAPHIE}

AmINZADE, R., et D. McAdAm (2001). «Emotions and contentious politics », dans R. AminZADE et al., Silence and Voice in the Study of Contentious Politics, New York, Cambridge University Press, p. 14-50.

Benford, R.D., et D.A. Snow (2000). « Framing Processes and Social Movement: An Overview and Assessment », Annual Review of Sociology, vol. 26, $\mathrm{n}^{\circ}$ 1, p. 611-639.

Calhoun, C. (2001). "Putting Emotions in their Place », dans J. GoodWIN, M. JASPER et F. PoletTA (dir.), Passionate Politics. Emotions and Social Movements, Chicago, University of Chicago Press, p. 45-58.

CANEL, E. (1992). «New social movement theory and resource mobilisation: the need for integration », dans W.K. CARROLL (dir.), Organizing Dissent, Toronto, Garamond, p. 22-51.

Damasio, A. (2006). L'erreur de Descartes. La raison des émotions, Paris, Odile Jacob, $2^{\mathrm{e}}$ édition.

DenZIN, N.K., et Y. LINCOLN (1998). Strategies of Qualitative Inquiry, Thousands Oaks, Sage.

DESLAURIERS, J.-P. (1991). Recherche qualitative : guide pratique, Toronto, MC Graw Hill.

DESLAURIERS, J.-P., et M. KÉRÉSIT (1997). «Le devis de recherche qualitative » dans J. POUPART et al., La recherche qualitative. Enjeux épistémologiques et méthodologiques, Boucherville, Gaëtan Morin, p. 85-111.

DobBIN, F. (2001). "The business of social movements », dans J. GoodWIN, J.M. JASPER, et F. Poletta (dir.), Passionate Politics. Emotions and Social Movements, Chicago, University of Chicago Press, p. 74-80.

DUPERRÉ, M. (2002). Constitution des acteurs collectifs et dynamique de développement régional: le cas d'une association régionale en santé et services sociaux, Université du Québec à Chicoutimi, Chicoutimi.

GidDENS, A. (1987). La constitution de la société, Paris, Presses universitaires de France.

GoodWin, J., J.M. JASPER, et F. PolletTA (2001). «Why Emotions Matter"», dans J. Goodwin, J.M. JASPER et F. PolletTA (dir.), Passionate Politics. Emotions and Social Movements, Chicago and London, The University of Chicago Press, p. 1-24.

GouLD, D.B. (2004). « Passionate Political Processes: Bringing Emotions Back into the Study of Social Movements », dans J. Goodwin et J.M. JASPER (dir.), Rethinking social movements, New York, Rowman and Littlefield publishers, p. 155-174.

Havard-Duclos, B., et S. Nicours (dir.). (2005). Pourquoi s'engager? Bénévoles et militants dans les associations de solidarité, Paris, Payot.

Huberman, M., et M. Miles, (1991). Analyse des données qualitatives : recueil de nouvelles méthodes, Bruxelles, Université De Bœck.

JASPER, J.M. (1998). «The Emotion of Protest: Affective and Reactive Emotions in and around Social Movements », Sociological Forum, vol. 13, n 3, p. 397-424.

Lamoureux, H., J. Lavoie, R. Mayer, et J. Panet-Raymond (2002). La pratique de l'action communautaire, Sainte-Foy, Presses de l'Université du Québec.

LOFLAND, J., et L. LOFLAND (1984). Analysing Social Settings, Belmont, California, Wadsworth. 
MarX-Ferree, M., et D.A. Merril (2004). « Hot movements, cold cognition: Thinking about social movements in gendered frames », dans J. GoODWIN et J.M. JASPER (dir.), Rethinking social movements, Toronto, Rowman and Littlefield Publ. p. 247-261.

Meluccl, A. (1994). "The Process of Collective Identity », dans H. JoHnston et B. KLANDERMANS (dir.), Social Movements and Culture, Minneapolis, University of Minnesota Press, p. 41-63.

NeVeu, É. (1996). Sociologie des mouvements sociaux, Paris, La Découverte. 